\title{
Six-Month Follow-Up Study of Ultrarapid Opiate Detoxification With Naltrexone
}

\author{
Mohammad Forozeshfard ${ }^{1}$; Babak Hosseinzadeh Zoroufchi ${ }^{1,}$; Mohammad Bagher Saberi \\ Zafarghandi ${ }^{2}$; Razieh Bandari ${ }^{3}$; Behzad Foroutan ${ }^{4}$ \\ ${ }^{1}$ Department of Anesthesia and Critical Care, Semnan University of Medical Sciences, Semnan, IR Iran \\ ${ }^{2}$ Department of Psychiatry, Tehran Psychiatric Institute, Tehran University of Medical Sciences, Tehran, IR Iran \\ ${ }^{3}$ Research Center for Social Determinants of Health, Semnan University of Medical Sciences, Semnan, IR Iran \\ 4 TDepartment of Pharmacology, School of Medicine, Shahroud University of Medical Sciences, Shahroud, IR Iran \\ *Corresponding author: Babak Hosseinzadeh Zoroufchi, Department of Anesthesia and Critical Care, Amir Hospital, Semnan University of Medical Sciences, Semnan, IR Iran. Tell: \\ +98-9121481098, Fax: +98-2333437849, E-mail: babakhz.anesthesia@gmail.com
}

Received: June 3, 2014; Revised: July 20, 2014; Accepted: August 3, 2014

\begin{abstract}
Background: Illicit opiate use has an increasing incidence and prevalence, which increases mortality and morbidity, marginalization, and criminal behaviors, and causes major adverse effects on society.

Objectives: This study aimed to investigate and follow the outcome of patients who underwent ultrarapid opiate detoxification (UROD) prospectively.

Patients and Methods: In this randomized clinical trial, 64 patients who underwent UROD were evaluated. The opiate antagonist regimen of naloxone was administered intravenously under general anesthesia, and detoxification was confirmed by naloxone challenge test. All patients were cared in intensive care unit (ICU) for 24 hours, and oral naltrexone was prescribed the next day, after recovery and discharge. Patients were followed up for one month after the procedure. Relapse was considered if routine use of opiates (daily use for at least two weeks) was reported by the patient after detoxification. The data was analyzed by SPSS 16.5 and the study was performed using descriptive analysis and Chi square test.

Results: All 64 participants were opiate-dependent males (ASA physical status of I or II) who aged over 18 years with a mean age of 31.11 \pm 8.93 years at the time of UROD. One month after UROD, 48 patients (75\%) reported relapse and 16 (25\%) reported abstinence; however, four patients of the non-relapsed group reported one episode of opiate use. There was no significant difference between relapsed and nonrelapsed patients regarding their marital status, level of education, and family history of opiate dependency $(\mathrm{P}>0.05)$.

Conclusions: Although UROD by naloxone is a safe and effective method of detoxification, if used alone, it has a very high relapse rate in long term.
\end{abstract}

Keywords: Opioid-Related Disorders; Metabolic Detoxification, Drug; Naloxone; Naltrexone; Recurrence

\section{Background}

Illicit opiate use has an increasing incidence and prevalence (1), which increases mortality and morbidity, marginalization, and criminal behaviors and causes major adverse effects on society (2). Consequently, achieving an effective therapeutic method for opiate dependency and addiction has become a focus of a variety of medical and legal professionals' attention (1). Over decades, strictly scheduled treatment with an opiate agonist such as methadone has been considered as the gold standard of therapy for opiate addiction (3). Due to the frequent failure and early relapse of conventional methods (4), some alternative nonopioid treatment techniques have been introduced, which involve oral or intravenous administration of opiate receptor antagonists (e.g. naloxone, and/or naltrexone) under deep sedation or general anesthesia for detoxification (3, 5-7). One of these techniques is ultrarapid opiate detoxification (UROD), which has become increasingly popular (5). It was claimed that UROD leads to complete cure for opiate addiction; therefore, this method has gained attention of both media and clinicians (8). In comparison to other detoxification methods, UROD has been reported as a promising method in the management of withdrawal syndrome and has markedly shortened withdrawal period (9). Although a large number of patients have been treated with UROD worldwide, there are still little scientific data on efficacy and long-term outcome of this procedure $(3,10)$. Opioid dependence is a chronic disorder and its prognosis is affected by a variety of factors. Patients' adherence to behavioral modification programs plays more important role in the prognosis of the opioid dependence treatment than detoxification per se. In order to provide a reliable evaluation of UROD efficacy, long-term follow-up is essential (3).

\section{Objectives}

The study was designed to investigate and follow the

Copyright (C) 2014, Zahedan University of Medical Sciences; Published by Kowsar. This is an open-access article distributed under the terms of the Creative Commons Attribution-NonCommercial 4.0 International License (http://creativecommons.org/licenses/by-nc/4.0/) which permits copy and redistribute the material just in noncommercial usages, provided the original work is properly cited. 
Forozeshfard M et al.

outcome of patients who underwent UROD, prospectively.

\section{Patients and Methods}

\subsection{Study Population and Design}

This was a prospective follow-up study. After obtaining the approval of the Ethics Committee of Semnan University of Medical Sciences and obtaining informed consent from participants, this prospective study was performed on 64 over 18-year-old opiate-dependent patients (ASA physical status of I or II), who were referred for UROD to Amir-al-Momenin Hospital, Semnan, Iran, between September 2011 and March 2012. Diagnosis of opiate dependency was confirmed by a psychiatrist according to diagnostic and statistical manual of mental disorders, 4th edition, text revision (DSM-IV-TR) (11). Patients who did not meet criteria, were unmotivated, had severe psychiatric disorders such as schizophrenia or bipolar disorder, or those who used other treatments for addiction simultaneously, were excluded. At the beginning of the study, patients were instructed to answer a checklist regarding demographic data, socioeconomic status, past medical history, and opiate dependency history.

\subsection{UROD Procedure}

On pre-UROD visit, procedure was explained to patients. Then all patients underwent a physical examination, an electrocardiogram, and biochemical blood tests including complete blood cells count, electrolytes, urea, creatinine, and transaminases. After 8 to 12 hours of fasting, patients were admitted to the hospital. An anesthesiologist and an anesthesiology technician closely observed vital signs during the procedure. Patients were premedicated with oral $5-\mu \mathrm{g} / \mathrm{kg}$ clonidine 90 minutes before the induction of general anesthesia. Then, based on patient's condition, general anesthesia was induced and maintained for four to six hours by isoflurane, atracurium, propofol, midazolam, or $\mathrm{NO}_{2}$ in doses dictated by patient response, and intubation followed. Patients were carefully observed for any changes in hemodynamic, vital signs, and withdrawal signs during anesthesia by a nurse and an anesthesiologist. Ventilatory frequency, $\mathrm{SpO}_{2}$, heart rate, ECG, blood pressure, and urine output were monitored continuously and recorded before and during the anesthesia. The opiate antagonist regimen of naloxone was administered intravenously. Detoxification was confirmed by naloxone challenge test. If the test failed to elicit withdrawal symptoms, UROD was considered to be complete and was terminated. Then, anesthesia was discontinued, patients were extubated, and kept in ICU for 24 hours. After recovery, patients received appropriate supportive and symptomatic treatments as needed. Oral naltrexone (50 mg) was prescribed the day after the procedure. If there was no major complication, patients were discharged on naltrexone (100-50 mg, daily), clonidine (0.2 $\mathrm{mg}$, daily), naproxen (500 mg, thrice a day), diazepam (5-10 mg, twice a day), and hyoscine (10 mg, twice a day). All patients were referred to a psychiatrist for free-ofcharge monthly visit.

\subsection{Follow-up}

Patients were followed up by attending in the clinic or by telephone one month after the procedure. During telephone interview, patients and first degree family members were asked about patient use of opiates or other drugs. If there was a history of relapse, date of first reuse was recorded. For patients with non-relapse, another interview was arranged three and six months after UROD to re-evaluate abstinence status. In order to validate patients responses, their responses were compared to significant others' answers. If any discrepancy was found, further attempts were made to find the reliable answer. According to previous studies, relapse was considered if interview confirmed routine use of opiates, ie, daily use for at least two weeks, by the patient after detoxification. If the patient or a significant other reported no return to routine opiate use, despite having episodes of opiate use since detoxification, it would be considered as nonrelapse (12-14).

Moreover, patients were asked about any additional treatment other than the study program, and if the answer was positive, they were excluded from the investigation. Data were analyzed by SPSS version 16.5 (SPSS Inc., Chicago, IL, USA) using descriptive analysis, independentsamples t-test and Chi-square test. $\mathrm{P}$ values $<0.05$ was considered statistically significant.

\section{Results}

\subsection{Baseline and Demographic Data}

All patients were opium-dependent males with a mean age of $31.11 \pm 8.93$ years at the time of UROD (range, 18-53). Two patients (3\%) were illiterate, 33 (52\%) had elementary school education level, and 29 (45\%) had high school diploma. None of them had university education. Regarding marital status, 24 patients (37\%) were single, 37 (58\%) were married, and 3 (5\%) were separated. None of them was divorced. History of opiate dependency in first-degree relatives was positive in 17 patients (26\%). In addition, 62 patients (97\%) were addicted to different types of opiates, and 2 (3\%) were addicted to Norgesic (Orphenadrine/Aspirin/Caffeine). Four patients (6\%) were simultaneously alcohol dependent. The main routes of substance abuse were as follows: oral route in 31 patients (49\%), smoking in 26 (41\%), and injection in 7 (10\%). None of patients had a history of high-risk sexual behaviors or using common syringes. Eight patients (12\%) had started opiate before the age of 16, 35 (55\%) between 16 and 20, 18 (28\%) between 20 and 30, and 3 (5\%) after the age of 30 years. Forty-six patients (71\%) had a previous history of addiction treatment; 29 patients (45\%) once, 15 (23\%) twice, 
and two (3\%) more than twice. According to the past history of detoxification, 45 patients (70\%) had experienced another detoxification method prior to UROD while 19 (30\%) were referred for detoxification for the first time. Among patients with positive history of detoxification, 41 (64\%) had undergone detoxification once and 22 (34\%) had undergone twice. One patient (1.5\%) reported history of detoxification for six times.

\subsection{UROD Adverse Effects}

All patients were asked about both physical and psychologic problems after the procedure. Physical discomfort was reported by 48 patients (75\%), while 53 (64\%) had psychologic problems. Physical discomfort included 12 cases of nausea (19\%), 27 cases of vomiting (42\%), five cases of diarrhea (8\%), three cases of abdominal pain (4\%), and 7 cases of muscular pain (11\%); some patients had more than one physical problem. Four patients (6\%) developed life-threatening problems including one case of pulmonary edema (1.5\%), one case of pneumothorax (1.5\%), one case of bradycardia (1.5\%), and one case of refractory delirium accompanied with high blood pressure and cardiac arrhythmia (1.5\%). All aforementioned cases were managed according to standard treatments. Psychological problems were consisted of agitation in 55 cases (86\%) and insomnia in ten patients (16\%).

\subsection{Follow-Up and Ultrarapid Opiate Detoxifica- tion Outcome}

All patients were alive and were called for follow-up interview; however, only 17 patients (27\%) attended in the clinic for interview. Therefore, a telephone interview was arranged for the remaining 47 patients (73\%). One month after UROD, 48 patients (75\%) reported relapse, and 16 (25\%) reported abstinence; however, four patients of the non-relapse group reported one episode of opiate use. There was no significant difference between relapsed and non-relapsed patients regarding marital status, family history of opiate dependency, and level of education (Table 1 ).

Among 16 patients of the non-relapse group, 12 patients relapsed three months after UROD and 4 patients reported relapse on the next interview six months after the procedure. Consequently, all patients relapsed six months after UROD. Mean of abstinence time was $37.34 \pm 21.03$ days since UROD. Only 25 patients (39\%) attended counseling sessions; however, none of them attended on a regular basis. There was no significant difference in mean of abstinence time between patients who attended counseling irregularly and those who did not attend at all (43.00 \pm 44.22 days and $33.65 \pm 30.75$ days, respectively; $\mathrm{P}=0.71$ ).

\begin{tabular}{|c|c|c|c|}
\hline & Relapsed & Non-Relapsed & P value \\
\hline Marital status & & & 0.42 \\
\hline Single & $19(40)$ & $5(31)$ & \\
\hline Married & $25(52)$ & $11(69)$ & \\
\hline Separated & $4(8)$ & 0 & \\
\hline Family History of Opiate Dependency & & & 0.10 \\
\hline Negative & $32(67)$ & $14(87)$ & \\
\hline Positive & $16(33)$ & $2(13)$ & \\
\hline Level of Education & & & 0.35 \\
\hline Illiterate & $2(4)$ & 0 & \\
\hline Elementary & $22(46)$ & $11(68)$ & \\
\hline High school & $24(50)$ & $5(32)$ & \\
\hline
\end{tabular}

\section{Discussion}

Opioid dependence is a complex illness and its treatment has been a problem for both patients and physicians (15). Different therapeutic methods such as compressed opiate detoxification (COD), naltrexonecompressed opiate detoxification (NCOD), rapid opiate detoxification (ROD) and UROD have emerged over years to achieve faster and more effective detoxification (16, 17). Prolonged detoxification programs frighten many opiate-dependent patients and therefore, they prefer accelerated detoxification programs. For this reason, short duration methods such as UROD have become more interesting for these patients (17). Opiate antagonists such as naloxone or naltrexone have been considered as an appropriate medication for UROD. This treatment has several useful and desirable features. Unlike opiate agonists, naloxone and naltrexone have no risk of misuse or 
legislative restrictions. In addition, UROD is remarkably safe, even in cases of overdose, and does not cause serious organ toxicity (18).

Despite many claims about the efficacy and usefulness of UROD, relapse rate is still the major concern of both patients and medical professions. This study showed that six months after UROD, all patients relapsed and restarted using opiates routinely. Majority of relapses occurred within the first month after the procedure. Although free counseling was provided for all patients after UROD, they did not attend counseling regularly, which could contribute to the high rate of UROD failure. Similar to our findings, Bochud et al. reported a high relapse rate of $88 \%$. In terms of long-term treatment, they concluded that UROD could be considered as a possible part of opiate treatment, but not an effective treatment per se (19).

In addition, another investigation by Favrat et al. demonstrated that 70\% of patients had relapsed three months after the opioid antagonist detoxification under anesthesia (20). In contrast, a study by Albanese et al. showed that only $45 \%$ of patients had relapsed six months after UROD (21); this lower relapse rate could be attributed to regular aftercare programs and naltrexone maintenance therapy (21).

Hensel et al. showed that UROD followed by long-term naltrexone maintenance program as well as a supportive psychotherapy could result in high rate of abstinence (68\%) 12 months after the procedure (22). Similarly, according to the findings of Rabinowitz et al. UROD combined with naltrexone maintenance and regular counseling resulted in a relapse rate of $43 \%$, which is lower than our rate. It confirmed useful role of combining UROD with other therapeutic methods (12).

In study of Teimori et al. on 104 patients, $87 \%$ of patients did not have drug dependency at the end of six months (23). In a more recent study by the same research team, they reiterated the importance of naltrexone maintenance and regular counseling following UROD in the prevention of relapse (24). UROD is a safe and efficient method, which successfully suppresses withdrawal symptoms and helps patients to tolerate acute phase better (22).

However, in light of the above evidence and findings of the current study, it can be inferred that in contrast to what many patients may believe, UROD itself is not the definite treatment of opiate dependency and it should be considered as a primary step in the long way of opiate dependency treatment. It means that UROD helps us to prepare patients more effectively as well as more rapidly for next steps of treatment. Following UROD by regular maintenance therapy and counseling might improve success rate of UROD in long term. However, in order to prove this hypothesis, we need further studies that compare relapse rate of UROD plus maintenance therapy and counseling with UROD alone in similar populations. In summary, although UROD by naloxone is a safe and effective method of detoxification, if it is used alone, it has a very high relapse rate in long term.

\section{Acknowledgements}

The authors are grateful to operation room and critical care nursing of Amir-Al-Momenin Hospital for their cooperation.

\section{Authors' Contributions}

Mohammad Forozeshfard, designing the study and preparation of the manuscript draft; Babak Hosseinzadeh Zoroufchi, preparing and editing the manuscript draft; Babak Hosseinzadeh Zoroufchi, conducting the study; Babak Hosseinzadeh Zoroufchi, Mohammad Forozeshfard, and Behzad Foroutan conducting the study and collecting data, Mohammad Bagher Saberi Zafarghandi, analysis of the data and interpreting the results; Razieh Bandari, preparing the manuscript and editing the final version.

\section{Funding/Support}

The presented research was funded by grants for thesis of Semnan University of Medical Sciences, Semnan, Iran.

\section{References}

1. Kaye AD, Gevirtz C, Bosscher HA, Duke JB, Frost EA, Richards TA et al. Ultrarapid opiate detoxification: a review. Can J Anaesth. 2003;50(7):663-71.

2. Lobmaier P, Gossop M, Waal H, Bramness J. The pharmacological treatment of opioid addiction--a clinical perspective. Eur J Clin Pharmacol. 2010;66(6):537-45.

3. Elman I, D'Ambra MN, Krause S, Breiter H, Kane M, Morris R, et al. Ultrarapid opioid detoxification: effects on cardiopulmonary physiology, stress hormones and clinical outcomes. Drug Alcohol Depend. 2001;61(2):163-72.

4. Ma H, Tang J, White PF, Wender RH, Leverone T, Quon R, et al. The effect of clonidine on gastrointestinal side effects associated with ultra-rapid opioid detoxification. Anesth Analg. 2003;96(5):140912 .

5. O'Connor PG, Kosten TR. Rapid and ultrarapid opioid detoxification techniques. JAMA.1998;279(3):229-34.

6. Lorenzi P, Marsili M, Boncinelli S, Fabbri LP, Fontanari P, Zorn AM et al. Searching for a general anaesthesia protocol for rapid detoxification from opioids. Eur J Anaesthesiol.1999;16(10):719-27.

7. Ward J, Hall W, Mattick RP. Role of maintenance treatment in opioid dependence. Lancet. 1999;353(9148):221-6.

8. Lawental E. Ultra rapid opiate detoxification as compared to 30 day inpatient detoxification program--a retrospective follow-up study. J Subst Abuse. 2000;11(2):173-81.

9. Kaye AD, Banister RE, Hoover JM, Baluch AR, Jacobs S, Shah RV Chronic pain and ultrarapid opioid detoxification. Pain Pract. 2005;5(1):33-42.

10. Strang J, Bearn J, Gossop M. Opiate detoxification under anaesthesia. BMJ.1997;315(7118):1249-50.

11. American Psychiatric Association. Diagnostic and statistical manual of mental disorders. Washington: DC; 2000.

12. Rabinowitz J, Cohen H, Tarrasch R, Kotler M. Compliance to naltrexone treatment after ultra-rapid opiate detoxification: an open label naturalistic study. Drug Alcohol Depend.1997;47(2):77-86.

13. Hubbard RL, Marsden ME. Relapse to use of heroin, cocaine, and other drugs in the first year after treatment. NIDA Res Monogr. 1986;72:157-66.

14. Unnithan S, Gossop M, Strang J. Factors associated with relapse 


\section{Forozeshfard M et al.}

among opiate addicts in an out-patient detoxification programme. BrJ Psychiatry. 1992;161:654-7.

15. Jaffe JH. Opioid related disorders. In: Sadock BJ editor. Comprehensive Textbook of Psychiatry. New York: Lippincott Williams and Wilkins; 2000.

16. Tretter F, Burkhardt D, Bussello-Spieth B, Reiss J, Walcher S, Buchele W. Clinical experience with antagonist-induced opiate withdrawal under anaesthesia. Addiction.1998;93(2):269-75.

17. Singh J, Basu D. Ultra-rapid opioid detoxification: current status and controversies. J Postgrad Med. 2004;50(3):227-32.

18. Brewer C, Streel E. Current Issues in the Use of Opioid Antagonists (Naltrexone for Opiate Abuse: A Re-Educational Tool as Well as an Effective Drug. In: Dean R, Bilsky E, Negus SS editors. Opiate Receptors and Antagonists. Humana; 2009.

19. Bochud Tornay C, Favrat B, Monnat M, Daeppen JB, Schnyder C, Bertschy G, et al. Ultra-rapid opiate detoxification using deep sedation and prior oral buprenorphine preparation: long-term results. Drug Alcohol Depend. 2003;69(3):283-8.
20. Favrat B, Zimmermann G, Zullino D, Krenz S, Dorogy F, Muller J, et al. Opioid antagonist detoxification under anaesthesia versus traditional clonidine detoxification combined with an additional week of psychosocial support: a randomised clinical trial. Drug Alcohol Depend. 2006;81(2):109-16.

21. Albanese AP, Gevirtz C, Oppenheim B, Field JM, Abels I, Eustace JC. Outcome and six month follow up of patients after Ultra Rapid Opiate Detoxification (UROD). J Addict Dis. 2000;19(2):11-28.

22. Hensel M, Kox WJ. Safety, efficacy, and long-term results of a modified version of rapid opiate detoxification under general anaesthesia: a prospective study in methadone, heroin, codeine and morphine addicts. Acta Anaesthesiol Scand. 2000;44(3):326-33.

23. Teimori H, Sabzi F, Tarrahi MJ, Heidari pour E. The success of this method of ultra rapid opiate detoxification (UROD) under general anesthesia. Yafte. 2008;11(1):11-6.

24. Rabinowitz J, Cohen H, Atias S. Outcomes of naltrexone maintenance following ultra rapid opiate detoxification versus intensive inpatient detoxification. Am J Addict. 2002;11(1):52-6. 